\title{
Partial Correctness of a Power Algorithm
}

\author{
Adrian Jaszczak \\ Institute of Informatics \\ University of Białystok \\ Poland
}

Summary. This work continues a formal verification of algorithms written in terms of simple-named complex-valued nominative data [6], [8, [15], 11, [12, ,13]. In this paper we present a formalization in the Mizar system [3] , 1 of the partial correctness of the algorithm:

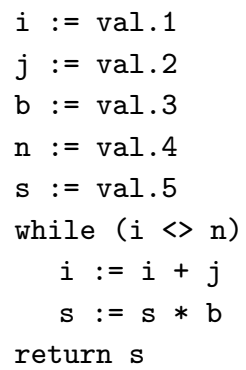

computing the natural $\mathrm{n}$ power of given complex number $\mathrm{b}$, where variables $\mathrm{i}$, $\mathrm{b}, \mathrm{n}, \mathrm{s}$ are located as values of a V-valued Function, loc, as: loc/.1 = i, loc $/ .3=\mathrm{b}, 10 \mathrm{c} / .4=\mathrm{n}$ and $\mathrm{loc} / .5=\mathrm{s}$, and the constant 1 is located in the location loc/.2 $=\mathrm{j}$ (set $\mathrm{V}$ represents simple names of considered nominative data [17).

The validity of the algorithm is presented in terms of semantic Floyd-Hoare triples over such data [9. Proofs of the correctness are based on an inference system for an extended Floyd-Hoare logic [2, 4, with partial pre- and post-conditions [14, [16, [7, [5].

\begin{tabular}{ll|l|l|l} 
MSC: 68Q60 & $68 \mathrm{~T} 37$ & $03 \mathrm{~B} 70$ & $03 \mathrm{~B} 35$ \\
\hline
\end{tabular}

Keywords: power; nominative data; program verification MML identifier: NOMIN_6, version: 8.1.09 5.57.1355 
Let $D$ be a set and $f_{1}, f_{2}, f_{3}, f_{4}, f_{5}$ be binominative functions of $D$. The functor PP-composition $\left(f_{1}, f_{2}, f_{3}, f_{4}, f_{5}\right)$ yielding a binominative function of $D$ is defined by the term

(Def. 1) PP-composition $\left(f_{1}, f_{2}, f_{3}, f_{4}\right) \bullet f_{5}$.

From now on $D$ denotes a non empty set, $f_{1}, f_{2}, f_{3}, f_{4}, f_{5}$ denote binominative functions of $D$, and $p, q, r, t, w, u$ denote partial predicates of $D$.

Now we state the proposition:

(1) Unconditional COMPosition RUle For 5 PRograms:

Suppose $\left\langle p, f_{1}, q\right\rangle$ is an SFHT of $D$ and $\left\langle q, f_{2}, r\right\rangle$ is an SFHT of $D$ and $\left\langle r, f_{3}, w\right\rangle$ is an SFHT of $D$ and $\left\langle w, f_{4}, t\right\rangle$ is an SFHT of $D$ and $\left\langle t, f_{5}, u\right\rangle$ is an SFHT of $D$ and $\left\langle\sim q, f_{2}, r\right\rangle$ is an SFHT of $D$ and $\left\langle\sim r, f_{3}, w\right\rangle$ is an SFHT of $D$ and $\left\langle\sim w, f_{4}, t\right\rangle$ is an SFHT of $D$ and $\left\langle\sim t, f_{5}, u\right\rangle$ is an SFHT of $D$. Then $\left\langle p, \mathrm{PP}\right.$-composition $\left.\left(f_{1}, f_{2}, f_{3}, f_{4}, f_{5}\right), u\right\rangle$ is an SFHT of $D$.

In the sequel $d, v, v_{1}$ denote objects, $V, A$ denote sets, $i, j, b, n, s, z$ denote elements of $V, i_{1}, j_{1}, b_{1}, n_{1}, s_{1}$ denote objects, $d_{1}, L_{2}, L_{3}, L_{1}, L_{4}, L_{5}$ denote non-atomic nominative data of $V$ and $A$, and $D_{2}, D_{3}, D_{1}, D_{4}, D_{5}$ denote binominative functions over simple-named complex-valued nominative date of $V$ and $A$.

Now we state the propositions:

(2) Suppose $V$ is not empty and $V$ is without nonatomic nominative data w.r.t. $A$ and $D_{2}=i_{1} \Rightarrow_{a}$ and $D_{3}=j_{1} \Rightarrow_{a}$ and $D_{1}=b_{1} \Rightarrow_{a}$ and $D_{4}=$ $n_{1} \Rightarrow_{a}$ and $D_{5}=s_{1} \Rightarrow_{a}$ and $L_{2}=d_{1} \nabla_{a}^{i} D_{2}\left(d_{1}\right)$ and $L_{3}=L_{2} \nabla_{a}^{j} D_{3}\left(L_{2}\right)$ and $L_{1}=L_{3} \nabla_{a}^{b} D_{1}\left(L_{3}\right)$ and $L_{4}=L_{1} \nabla_{a}^{n} D_{4}\left(L_{1}\right)$ and $L_{5}=L_{4} \nabla_{a}^{s} D_{5}\left(L_{4}\right)$ and $j_{1}$, $b_{1}, n_{1}, s_{1} \in \operatorname{dom} d_{1}$ and $d_{1} \in \operatorname{dom} D_{2}$ and $s \neq n$. Then $L_{5}(n)=L_{4}(n)$.

(3) Suppose $V$ is not empty and $V$ is without nonatomic nominative data w.r.t. $A$ and $D_{2}=i_{1} \Rightarrow_{a}$ and $D_{3}=j_{1} \Rightarrow_{a}$ and $D_{1}=b_{1} \Rightarrow_{a}$ and $D_{4}=$ $n_{1} \Rightarrow_{a}$ and $D_{5}=s_{1} \Rightarrow_{a}$ and $L_{2}=d_{1} \nabla_{a}^{i} D_{2}\left(d_{1}\right)$ and $L_{3}=L_{2} \nabla_{a}^{j} D_{3}\left(L_{2}\right)$ and $L_{1}=L_{3} \nabla_{a}^{b} D_{1}\left(L_{3}\right)$ and $L_{4}=L_{1} \nabla_{a}^{n} D_{4}\left(L_{1}\right)$ and $L_{5}=L_{4} \nabla_{a}^{s} D_{5}\left(L_{4}\right)$ and $j_{1}$, $b_{1}, n_{1}, s_{1} \in \operatorname{dom} d_{1}$ and $d_{1} \in \operatorname{dom} D_{2}$ and $s \neq i$. Then $L_{5}(i)=L_{4}(i)$.

In the sequel $f$ denotes a binominative function over simple-named complexvalued nominative data of $V$ and $A, T$ denotes a nominative data with simple names from $V$ and complex values from $A$, loc denotes a $V$-valued function, and val denotes a function.

Let us consider $V, A$, and loc. The functor power-loop-body $(A, l o c)$ yielding a binominative function over simple-named complex-valued nominative data of $V$ and $A$ is defined by the term

(Def. 2) $\left.\operatorname{Asg}^{(l o c / 1}\right)(\operatorname{addition}(A, l o c / 1, l o c / 2)) \bullet\left(\operatorname{Asg}^{(l o c / 5}\right)(\operatorname{multiplication}(A, l o c / 5$, $\left.\left.\left.l o c_{/ 3}\right)\right)\right)$. 
The functor power-main-loop $(A, l o c)$ yielding a binominative function over simple-named complex-valued nominative data of $V$ and $A$ is defined by the term

(Def. 3) $\mathrm{WH}\left(\neg \operatorname{Equality}\left(A, \operatorname{loc}_{/ 1}, \operatorname{loc}_{/ 4}\right)\right.$, power-loop-body $\left.(A, l o c)\right)$.

Let us consider val. The functor power-var-init $(A, l o c, v a l)$ yielding a binominative function over simple-named complex-valued nominative data of $V$ and $A$ is defined by the term

(Def. 4) PP-composition( $\operatorname{Asg}^{\left(l^{(o c / 1}\right)}\left(\operatorname{val}(1) \Rightarrow_{a}\right), \operatorname{Asg}^{\left(l^{l o c} / 2\right)}\left(\operatorname{val}(2) \Rightarrow_{a}\right)$,

$$
\left.\left.\operatorname{Asg}^{(l o c / 3}\right)\left(\operatorname{val}(3) \Rightarrow_{a}\right), \operatorname{Asg}^{\left(l^{l o c} / 4\right)}\left(\operatorname{val}(4) \Rightarrow_{a}\right), \operatorname{Asg}^{(l o c / 5)}\left(\operatorname{val}(5) \Rightarrow_{a}\right)\right) \text {. }
$$

The functor power-main-part $(A, l o c, v a l)$ yielding a binominative function over simple-named complex-valued nominative data of $V$ and $A$ is defined by the term

(Def. 5) power-var-init $(A, l o c, v a l) \bullet($ power-main-loop $(A, l o c))$.

Let us consider $z$. The functor power-program $(A, l o c, v a l, z)$ yielding a binominative function over simple-named complex-valued nominative data of $V$ and $A$ is defined by the term

(Def. 6) power-main-part $(A, l o c, v a l) \bullet\left(\operatorname{Asg}^{z}\left((l o c / 5) \Rightarrow_{a}\right)\right)$.

In the sequel $n_{0}$ denotes a natural number and $b_{0}$ denotes a complex number.

Let us consider $V, A, v a l, b_{0}, n_{0}$, and $d$. We say that $b_{0}, n_{0}$ and $d$ constitute a valid input for the power w.r.t. $V, A$ and val if and only if

(Def. 7) there exists a non-atomic nominative data $d_{1}$ of $V$ and $A$ such that $d=$ $d_{1}$ and $\{\operatorname{val}(1), \operatorname{val}(2), \operatorname{val}(3), \operatorname{val}(4), \operatorname{val}(5)\} \subseteq \operatorname{dom} d_{1}$ and $d_{1}(\operatorname{val}(1))=$ 0 and $d_{1}(\operatorname{val}(2))=1$ and $d_{1}(\operatorname{val}(3))=b_{0}$ and $d_{1}(\operatorname{val}(4))=n_{0}$ and $d_{1}(\operatorname{val}(5))=1$.

The functor valid-power-input $\left(V, A, v a l, b_{0}, n_{0}\right)$ yielding a partial predicate over simple-named complex-valued nominative data of $V$ and $A$ is defined by

(Def. 8) $\operatorname{dom} i t=\mathrm{ND}_{\mathrm{SC}}(V, A)$ and for every object $d$ such that $d \in \operatorname{dom} i t$ holds if $b_{0}, n_{0}$ and $d$ constitute a valid input for the power w.r.t. $V, A$ and val, then $i t(d)=$ true and if $b_{0}, n_{0}$ and $d$ do not constitute a valid input for the power w.r.t. $V, A$ and val, then $i t(d)=$ false.

Let us observe that valid-power-input $\left(V, A, v a l, b_{0}, n_{0}\right)$ is total.

Let us consider $z$ and $d$. We say that $b_{0}, n_{0}$ and $d$ constitute a valid output for the power w.r.t. $A$ and $z$ if and only if

(Def. 9) there exists a non-atomic nominative data $d_{1}$ of $V$ and $A$ such that $d=d_{1}$ and $z \in \operatorname{dom} d_{1}$ and $d_{1}(z)=b_{0}{ }^{n_{0}}$.

The functor valid-power-output $\left(A, z, b_{0}, n_{0}\right)$ yielding a partial predicate over simple-named complex-valued nominative data of $V$ and $A$ is defined by 
(Def. 10) $\operatorname{dom} i t=\{d$, where $d$ is a nominative data with simple names from $V$ and complex values from $\left.A: d \in \operatorname{dom}\left(z \Rightarrow_{a}\right)\right\}$ and for every object $d$ such that $d \in \operatorname{dom} i t$ holds if $b_{0}, n_{0}$ and $d$ constitute a valid output for the power w.r.t. $A$ and $z$, then $i t(d)=$ true and if $b_{0}, n_{0}$ and $d$ do not constitute a valid output for the power w.r.t. $A$ and $z$, then $i t(d)=$ false.

Let us consider $l o c$ and $d$. We say that $b_{0}, n_{0}$ and $d$ constitute a valid invariant for the power w.r.t. $A$ and $l o c$ if and only if

(Def. 11) there exists a non-atomic nominative data $d_{1}$ of $V$ and $A$ such that $d=d_{1}$ and $\left\{l o c_{/ 1}, l o c_{/ 2}, l o c_{/ 3}, l o c_{/ 4}, l o c_{/ 5}\right\} \subseteq \operatorname{dom} d_{1}$ and $d_{1}\left(l o c_{/ 2}\right)=1$ and $d_{1}\left(l o c_{3}\right)=b_{0}$ and $d_{1}\left(l o c_{/ 4}\right)=n_{0}$ and there exists a complex number $S$ and there exists a natural number $I$ such that $I=d_{1}\left(l o c_{1}\right)$ and $S=d_{1}(l o c / 5)$ and $S=b_{0}{ }^{I}$.

The functor PP-composition $\left(A, l o c, b_{0}, n_{0}\right)$ yielding a partial predicate over simple-named complex-valued nominative data of $V$ and $A$ is defined by

(Def. 12) $\operatorname{dom} i t=\operatorname{ND}_{\mathrm{SC}}(V, A)$ and for every object $d$ such that $d \in \operatorname{dom} i t$ holds if $b_{0}, n_{0}$ and $d$ constitute a valid invariant for the power w.r.t. $A$ and $l o c$, then it $(d)=$ true and if $b_{0}, n_{0}$ and $d$ do not constitute a valid invariant for the power w.r.t. $A$ and $l o c$, then $i t(d)=$ false.

Observe that PP-composition $\left(A, l o c, b_{0}, n_{0}\right)$ is total.

Let us consider val. We say that $l o c$ and $v a l$ are compatible w.r.t. 5 locations if and only if

$\left(\right.$ Def. 13) $\operatorname{val}(5) \neq l o c_{/ 4}$ and $\operatorname{val}(5) \neq l o c_{/ 3}$ and $\operatorname{val}(5) \neq l o c_{/ 2}$ and $\operatorname{val}(5) \neq l o c_{/ 1}$ and $\operatorname{val}(4) \neq l o c_{/ 3}$ and $\operatorname{val}(4) \neq l o c_{/ 2}$ and $\operatorname{val}(4) \neq l o c_{/ 1}$ and $\operatorname{val}(3) \neq$ $l o c_{/ 2}$ and $\operatorname{val}(3) \neq l o c_{/ 1}$ and $\operatorname{val}(2) \neq l o c_{/ 1}$.

Now we state the propositions:

(4) Suppose $V$ is not empty and $V$ is without nonatomic nominative data w.r.t. $A$ and $l o c_{1}, l o c_{/ 2}, l o c_{/ 3}, l o c_{/ 4}, l o c_{/ 5}$ are mutually different and $l o c$ and val are compatible w.r.t. 5 locations. Then /valid-power-input $\left(V, A, v a l, b_{0}\right.$, $\left.n_{0}\right)$, power-var-init $(A, l o c, v a l), \mathrm{PP}$-composition $\left.\left(A, l o c, b_{0}, n_{0}\right)\right\rangle$ is an SFHT of $\operatorname{ND}_{\mathrm{SC}}(V, A)$.

Proof: Set $i=l o c_{/ 1}$. Set $j=l o c_{/ 2}$. Set $b=l o c_{/ 3}$. Set $n=l o c_{/ 4}$. Set $s=l o c / 5$. Set $i_{1}=\operatorname{val}(1)$. Set $j_{1}=\operatorname{val}(2)$. Set $b_{1}=\operatorname{val}(3)$. Set $n_{1}=$ $\operatorname{val}(4)$. Set $s_{1}=\operatorname{val}(5)$. Set $I=\operatorname{valid}-\operatorname{power-input}\left(V, A, \operatorname{val}, b_{0}, n_{0}\right)$. Set $i_{2}=$ PP-composition $\left(A, l o c, b_{0}, n_{0}\right)$. Set $D_{2}=i_{1} \Rightarrow_{a}$. Set $D_{3}=j_{1} \Rightarrow_{a}$. Set $D_{1}=b_{1} \Rightarrow_{a}$. Set $D_{4}=n_{1} \Rightarrow_{a}$. Set $D_{5}=s_{1} \Rightarrow_{a}$. Set $T_{1}=\mathrm{SP}_{\mathrm{P}}\left(i_{2}, D_{5}, s\right)$. Set $S_{1}=\mathrm{S}_{\mathrm{P}}\left(T_{1}, D_{4}, n\right)$. Set $R_{1}=\mathrm{S}_{\mathrm{P}}\left(S_{1}, D_{1}, b\right)$. Set $Q_{1}=\mathrm{S}_{\mathrm{P}}\left(R_{1}, D_{3}, j\right)$. Set $P_{1}=\mathrm{SP}_{\mathrm{P}}\left(Q_{1}, D_{2}, i\right) . I \models P_{1}$ by [6, (39)], [8, (9)], [10, (4)].

(5) Suppose $V$ is not empty and $A$ is complex containing and $V$ is without nonatomic nominative data w.r.t. $A$ and $l o c_{11}, l o c_{/ 2}, l o c_{/ 3}, l o c_{/ 4}, l o c / 5$ are 
mutually different. Then $\left\langle\mathrm{PP}\right.$-composition $\left(A, l o c, b_{0}, n_{0}\right)$, power-loop-body $(A, l o c), \mathrm{PP}$-composition $\left.\left(A, l o c, b_{0}, n_{0}\right)\right\rangle$ is an $\operatorname{SFHT}_{\text {of }} \mathrm{ND}_{\mathrm{SC}}(V, A)$.

(6) $\left\langle\sim \mathrm{PP}\right.$-composition $\left(A, l o c, b_{0}, n_{0}\right)$, power-loop-body $(A, l o c)$, $\mathrm{PP}$-composition $\left.\left(A, l o c, b_{0}, n_{0}\right)\right\rangle$ is an $\operatorname{SFHT}$ of $\mathrm{ND}_{\mathrm{SC}}(V, A)$.

(7) Suppose $V$ is not empty and $A$ is complex containing and $V$ is without nonatomic nominative data w.r.t. $A$ and $l o c / 1, l o c / 2, l o c / 3, l o c / 4, l o c / 5$ are mutually different. Then $\left\langle\mathrm{PP}\right.$-composition $\left(A, l o c, b_{0}, n_{0}\right)$, power-main-loop $(A, l o c), \operatorname{Equality}\left(A, l_{o c} / 1, l_{o c} / 4\right) \wedge \mathrm{PP}$-composition $\left.\left(A, l o c, b_{0}, n_{0}\right)\right\rangle$ is an SF$\mathrm{HT}$ of $\mathrm{ND}_{\mathrm{SC}}(V, A)$. The theorem is a consequence of (5) and (6).

(8) Suppose $V$ is not empty and $A$ is complex containing and $V$ is without nonatomic nominative data w.r.t. $A$ and $l o c_{1}, l_{o c} / 2, l o c / 3, l o c / 4, l o c / 5$ are mutually different and $l o c$ and $v a l$ are compatible w.r.t. 5 locations. Then 〈valid-power-input $\left(V, A, v a l, b_{0}, n_{0}\right)$, power-main-part $(A, l o c, v a l)$, Equality $(A, l o c / 1, l o c / 4) \wedge \mathrm{PP}$-composition $\left.\left(A, l o c, b_{0}, n_{0}\right)\right\rangle$ is an $\operatorname{SFHT}_{\text {of }} \mathrm{ND}_{\mathrm{SC}}(V, A)$. The theorem is a consequence of (4) and (7).

(9) Suppose $V$ is not empty and $V$ is without nonatomic nominative data w.r.t. $A$ and for every $T, T$ is a value on $l o c / 1$ and for every $T, T$ is a value on $l o c / 4$. Then $\operatorname{Equality}(A, l o c / 1, l o c / 4) \wedge \mathrm{PP}$-composition $\left(A, l o c, b_{0}, n_{0}\right) \models$ $\mathrm{S}_{\mathrm{P}}\left(\right.$ valid-power-output $\left.\left(A, z, b_{0}, n_{0}\right),(l o c / 5) \Rightarrow_{a}, z\right)$.

Proof: Set $i=l o c_{/ 1}$. Set $j=l_{o c} / 2$. Set $b=l o c_{/ 3}$. Set $n=l_{o c} / 4$. Set $s=l o c / 5$. Set $D_{5}=s \Rightarrow_{a}$. Consider $d_{1}$ being a non-atomic nominative data of $V$ and $A$ such that $d=d_{1}$ and $\{i, j, b, n, s\} \subseteq \operatorname{dom} d_{1}$ and $d_{1}(n)=n_{0}$ and $d_{1}(b)=b_{0}$ and there exists a complex number $S$ and there exists a natural number $I$ such that $I=d_{1}(i)$ and $S=d_{1}(s)$ and $S=b_{0}{ }^{I}$. Reconsider $d_{2}=d$ as a nominative data with simple names from $V$ and complex values from $A$. Set $L=d_{2} \nabla_{a}^{z} D_{5}\left(d_{2}\right) . b_{0}, n_{0}$ and $L$ constitute a valid output for the power w.r.t. $A$ and $z$.

(10) Suppose $V$ is not empty and $V$ is without nonatomic nominative data w.r.t. $A$ and for every $T, T$ is a value on $l o c / 1$ and for every $T, T$ is a value on $\operatorname{loc}_{/ 4}$. Then $\left\langle\operatorname{Equality}\left(A, \operatorname{loc}_{/ 1}, l_{o c} / 4\right) \wedge \mathrm{PP}\right.$-composition $\left(A, l o c, b_{0}, n_{0}\right)$, $\operatorname{Asg}^{z}\left((l o c / 5) \Rightarrow_{a}\right)$, valid-power-output $\left.\left(A, z, b_{0}, n_{0}\right)\right\rangle$ is an $\operatorname{SFHT}_{\text {of }} \mathrm{ND}_{\mathrm{SC}}(V$, $A)$. The theorem is a consequence of $(9)$.

(11) Suppose for every $T, T$ is a value on $l o c / 1$ and for every $T, T$ is a value on $\operatorname{loc}_{/ 4}$. Then $\left\langle\sim\left(\operatorname{Equality}\left(A, l_{o c} / 1, l_{o c} / 4\right) \wedge \mathrm{PP}\right.\right.$-composition $\left.\left(A, l o c, b_{0}, n_{0}\right)\right)$, $\operatorname{Asg}^{z}\left(\left(\operatorname{loc}_{/ 5}\right) \Rightarrow_{a}\right)$, valid-power-output $\left.\left(A, z, b_{0}, n_{0}\right)\right\rangle$ is an $\operatorname{SFHT}_{\text {of }} \mathrm{ND}_{\mathrm{SC}}(V, A)$.

(12) Partial Correctness of A POWER Algorithm:

Suppose $V$ is not empty and $A$ is complex containing and $V$ is without nonatomic nominative data w.r.t. $A$ and $l o c / 1, l o c / 2, l o c / 3, l o c / 4, l o c / 5$ are 
mutually different and $l o c$ and val are compatible w.r.t. 5 locations and for every $T, T$ is a value on $l o c_{/ 1}$ and for every $T, T$ is a value on $l o c_{/ 4}$. Then 〈valid-power-input $\left(V, A, v a l, b_{0}, n_{0}\right)$, power-program $(A, l o c, v a l, z)$, valid-power-output $\left.\left(A, z, b_{0}, n_{0}\right)\right\rangle$ is an $\operatorname{SFHT}$ of $\operatorname{ND}_{\mathrm{SC}}(V, A)$. The theorem is a consequence of $(8),(10)$, and (11).

\section{REFERENCES}

[1] Grzegorz Bancerek, Czesław Byliński, Adam Grabowski, Artur Korniłowicz, Roman Matuszewski, Adam Naumowicz, and Karol Pąk. The role of the Mizar Mathematical Library for interactive proof development in Mizar Journal of Automated Reasoning, 61(1):9-32, 2018. dol:10.1007/s10817-017-9440-6

[2] R.W. Floyd. Assigning meanings to programs. Mathematical aspects of computer science, 19(19-32), 1967.

[3] Adam Grabowski, Artur Korniłowicz, and Adam Naumowicz. Four decades of Mizar. Journal of Automated Reasoning, 55(3):191-198, 2015. doi 10.1007/s10817-015-9345-1

[4] C.A.R. Hoare. An axiomatic basis for computer programming. Commun. ACM, 12(10): 576-580, 1969.

[5] Ievgen Ivanov and Mykola Nikitchenko. On the sequence rule for the Floyd-Hoare logic with partial pre- and post-conditions. In Proceedings of the 14 th International Conference on ICT in Education, Research and Industrial Applications. Integration, Harmonization and Knowledge Transfer. Volume II: Workshops, Kyiv, Ukraine, May 14-17, 2018, volume 2104 of CEUR Workshop Proceedings, pages 716-724, 2018.

[6] Ievgen Ivanov, Mykola Nikitchenko, Andrii Kryvolap, and Artur Korniłowicz. Simplenamed complex-valued nominative data - definition and basic operations. Formalized Mathematics, 25(3):205-216, 2017. doi 10.1515/forma-2017-0020

[7] Ievgen Ivanov, Artur Korniłowicz, and Mykola Nikitchenko. Implementation of the composition-nominative approach to program formalization in Mizar. The Computer Science Journal of Moldova, 26(1):59-76, 2018.

[8] Ievgen Ivanov, Artur Korniłowicz, and Mykola Nikitchenko. On an algorithmic algebra over simple-named complex-valued nominative data. Formalized Mathematics, 26(2):149158, 2018. doi $10.2478 /$ forma-2018-0012.

[9] Ievgen Ivanov, Artur Korniłowicz, and Mykola Nikitchenko. An inference system of an extension of Floyd-Hoare logic for partial predicates. Formalized Mathematics, 26(2): 159-164, 2018. doi $10.2478 /$ forma-2018-0013.

[10] Ievgen Ivanov, Artur Korniłowicz, and Mykola Nikitchenko. Partial correctness of GCD algorithm. Formalized Mathematics, 26(2):165-173, 2018. doi 10.2478/forma-2018-0014.

[11] Ievgen Ivanov, Artur Korniłowicz, and Mykola Nikitchenko. On algebras of algorithms and specifications over uninterpreted data. Formalized Mathematics, 26(2):141-147, 2018. doi $10.2478 /$ forma-2018-0011.

[12] Artur Kornilowicz, Andrii Kryvolap, Mykola Nikitchenko, and Ievgen Ivanov. Formalization of the algebra of nominative data in Mizar In Maria Ganzha, Leszek A. Maciaszek, and Marcin Paprzycki, editors, Proceedings of the 2017 Federated Conference on Computer Science and Information Systems, FedCSIS 2017, Prague, Czech Republic, September 3-6, 2017., pages 237-244, 2017. ISBN 978-83-946253-7-5. doi $10.15439 / 2017$ F301.

[13] Artur Kornilowicz, Andrii Kryvolap, Mykola Nikitchenko, and Ievgen Ivanov. Formalization of the nominative algorithmic algebra in Mizar. In Leszek Borzemski, Jerzy Świątek, and Zofia Wilimowska, editors, Information Systems Architecture and Technology: Proceedings of 38th International Conference on Information Systems Architecture and Technology - ISAT 2017 - Part II, Szklarska Poręba, Poland, September 17-19, 2017, volume 656 of Advances in Intelligent Systems and Computinq, pages 176-186. Springer, 2017. ISBN 978-3-319-67228-1. doi 10.1007/978-3-319-67229-8_16

[14] Artur Korniłowicz, Andrii Kryvolap, Mykola Nikitchenko, and Ievgen Ivanov. An approach to formalization of an extension of Floyd-Hoare logic. In Vadim Ermolayev, Nick Bassiliades, Hans-Georg Fill, Vitaliy Yakovyna, Heinrich C. Mayr, Vyacheslav Kharchen- 
ko, Vladimir Peschanenko, Mariya Shyshkina, Mykola Nikitchenko, and Aleksander Spivakovsky, editors, Proceedings of the 13th International Conference on ICT in Education, Research and Industrial Applications. Integration, Harmonization and Knowledge Transfer, Kyiv, Ukraine, May 15-18, 2017, volume 1844 of CEUR Workshop Proceedings, pages 504-523. CEUR-WS.org, 2017.

[15] Artur Korniłowicz, Ievgen Ivanov, and Mykola Nikitchenko. Kleene algebra of partial predicates. Formalized Mathematics, 26(1):11-20, 2018. doi:10.2478/forma-2018-0002.

[16] Andrii Kryvolap, Mykola Nikitchenko, and Wolfgang Schreiner. Extending Floyd-Hoare logic for partial pre- and postconditions In Vadim Ermolayev, Heinrıch C. Mayr, Mykola Nikitchenko, Aleksander Spivakovsky, and Grygoriy Zholtkevych, editors, Information and Communication Technologies in Education, Research, and Industrial Applications: 9th International Conference, ICTERI 2013, Kherson, Ukraine, June 19-22, 2013, Revised Selected Papers, pages 355-378. Springer International Publishing, 2013. ISBN 978-3-31903998-5. doi 10.1007/978-3-319-03998-5_18.

[17] Volodymyr G. Skobelev, Mykola Nikitchenko, and Ievgen Ivanov. On algebraic properties of nominative data and functions. In Vadim Ermolayev, Heinrich C. Mayr, Mykola N1kitchenko, Aleksander Spivakovsky, and Grygoriy Zholtkevych, editors, Information and Communication Technologies in Education, Research, and Industrial Applications - 10th International Conference, ICTERI 2014, Kherson, Ukraine, June 9-12, 2014, Revised Selected Papers, volume 469 of Communications in Computer and Information Science, pages 117-138. Springer, 2014. ISBN 978-3-319-13205-1. doi $10.1007 / 978-3-319-13206-8 \_6$.

Accepted May 27, 2019 